\title{
Speeding Online Synthesis via Enforced Selecto-Recombination
}

\author{
Shunsuke Saruwatari ${ }^{1}$, Xavier Llorà ${ }^{1,2}$, Noriko Imafuji Yasui ${ }^{1}$, \\ Hiroshi Tamura ${ }^{3}$, Kumara Sastry ${ }^{1}$, and David E. Goldberg' ${ }^{1}$ \\ ${ }^{1}$ Illinois Genetic Algorithms Laboratory (IlliGAL), Dept. of Industrial and Enterprise Systems Eng. \\ University of Illinois at Urbana-Champaign, Urbana IL 61801 \\ ${ }^{2}$ National Center for Supercomputing Applications (NCSA) \\ University of Illinois at Urbana-Champaign, Urbana IL 61801 \\ ${ }^{3}$ Research and Development Division, Hakuhodo Inc, Tokyo 108-8088, Japan. \\ \{saru,xllora,niyasui,ksastry\}@uiuc.edu, hiroshi.tamura@hakuhodo.co.jp, \\ deg@uiuc.edu
}

\begin{abstract}
Brainstorming has been greatly used as a method to generate a large number of ideas by variety of each participant's knowledge. However, brainstorming does not always work well because of spatial and communication limitations. Moreover, brainstorming techniques present limited scalability. Meanwhile, genetic algorithms have been mostly regarded as an engineering or technological tool. However, the innovation intuition suggests that genetic algorithms may be also regarded as models of human innovation and creativity. This paper focuses on online creativity sessions. Modeling those creative efforts using selecto-recombinative mechanism can provide three times more novel ideas, increase the posting frequency by a 2.6 factor, and help overcome superficiality on online communications by favoring synthetic thinking.
\end{abstract}

\section{Categories and Subject Descriptors}

H.4.3 [Information Systems Applications]: Communications Applications; H.5.3 [Information Interfaces and Presentation]: Group and Organization Interfaces

\section{General Terms}

Algorithms, Design, Experimentation

\section{Keywords}

Innovation, creativity, brainstorming, online discussions, human-based genetic algorithms, superficiality on online communication

Permission to make digital or hard copies of all or part of this work for personal or classroom use is granted without fee provided that copies are not made or distributed for profit or commercial advantage and that copies bear this notice and the full citation on the first page. To copy otherwise, to republish, to post on servers or to redistribute to lists, requires prior specific permission and/or a fee.

GECCO'08, July 12-16, 2008, Atlanta, Georgia, USA.

Copyright 2008 ACM 978-1-60558-130-9/08/07...\$5.00.

\section{INTRODUCTION}

Genetic algorithms have become a widely used tool for problem solving in engineering and technology areas. The advances in competent genetic algorithms [6] have made possible to solve quickly, reliable, and accurately problems of unprecedented size [17]. However, genetic algorithms have also entered areas ruled by aesthetic criteria; interactive genetic algorithms [18] are a clear exponent of collaborative human-computer problem solving where no objective, but subjective, function can be defined. Moreover, social aspects of genetic algorithms have shown how they can act as models of human innovation and creativity [12, 8, 13, 20] - as postulated by Goldberg [5]. Human-based genetic algorithms (HBGAs) target human process by drawing from the lessons learned from their computational counterparts. Figure 1 summarizes the set of possible modes of cooperation between humans and computers by means of genetic algorithms.

This paper continues the exploration of the social aspects of genetic algorithms. Human-based genetic algorithms can be metaphors of organizations, but also models of human innovation and creativity. Early efforts have shown the benefits of modeling creative processes after the evolutionary metaphor $[8,13]$. However, those efforts lacked of quantitative analysis. In this paper, we focus on online creativity sessions. Usually mediated by some form of bulletin boards or threaded discussion boards (TDB), creativity can be boosted by modeling the online activities after a HBGA. Our work will compare traditional TDB and HBGA-enhanced one to provide a quantitative comparison of the benefits introduced by the evolutionary modeling. We will show how an online HBGA-based process can (1) provide three times more novel ideas, (2) increase the posting frequency by a 2.6 factor, and (3) help overcome superficiality on online communications by favoring synthetic thinking.

The rest of this paper is organized as follows. Section 2 describes the basic framework of the work presented in this paper. Section 3 presents and reviews the details of a traditional TDB and its HBGA-enhanced counter part. Then, section 4 presents the experimental setup, whereas section 5 analyzes the results obtained using both approaches and 


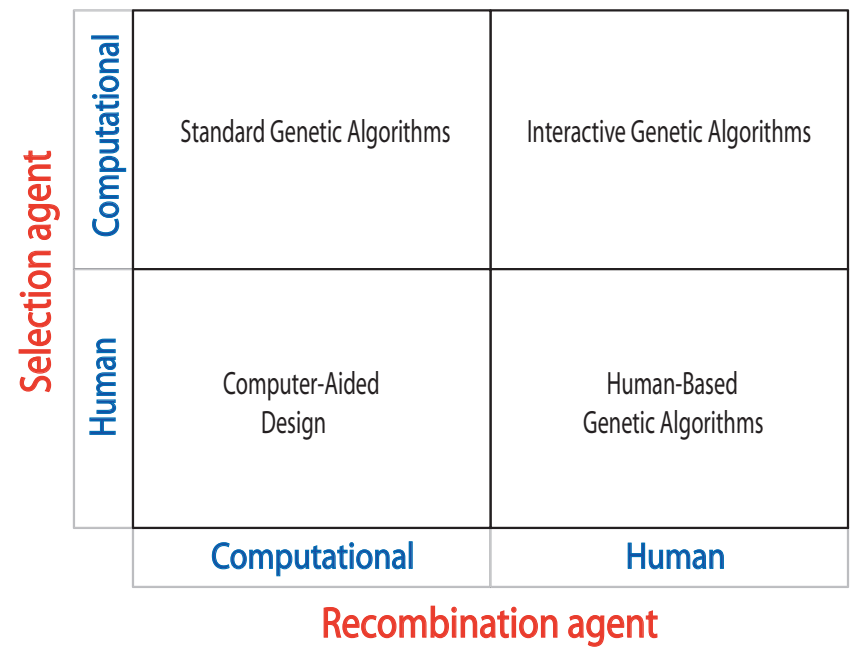

Figure 1: The human-computer interaction quadrant shows different modes of genetic algorithms as models of human innovation.

the improvements achieved by using the evolution-inspired TDB. The paper is concluded in section 6 .

\section{CREATIVITY, INNOVATION, AND THE ONLINE MEDIA}

Starting in 1983, Goldberg [5] developed the so called fundamental intuition of genetic algorithms, or the innovation intuition. Specifically, the innovation intuition of GAs is about the work together of: (1) selection and mutation, and (2) selection and recombination. Moreover, the innovation intuition of GAs provides a facet-wise modeling of human innovation. This approach models two orthogonal facets of human innovation.

Selection + mutation $=$ Continual improvement. Selection and mutation working together are a form of hill-climbing mechanism. Mutation suggests variants in the neighborhood of the current solutions; selection acts as the decision process which accepts improving changes with a high probability. This simple model describes one of the facets of human innovation, the so called continual improvement in total quality management literature, or as Japanese call it, kaizen.

Selection + crossover $=$ innovation. Another facet of human innovation is the so called cross-fertilizing innovation. People usually grasp a set of good solution features in one context, and a notion in another context and juxtaposing them, thereby speculating that the combination might be better than either notion taken individually. Taking together selection and crossover, GAs are a computation model of cross-fertilizing innovation.

GAs also are main role players for the innovation technology revolution. As early mentioned, humans are to become the main measure of such a technology. Pervasive GA-guided interaction between human and computers opens a new research path to creativity- and innovation-support. Two well-known models of such support are interactive GAs, and human-based GAs. Interactive GAs (iGAs) replace the computer computation of the relative fitness of solutions and the selection process by the judgment of a human evaluation. More detailed information about the progress of interactive GAs (iGAs) and interactive evolutionary computation (iEC) are presented in a review by Takagi [18]. Whereas iGAs replace the evaluation and selection by the human judgment, human-based GAs (HBGAs) [12] move one step further and permit evaluation, selection, and variation to be performed by a human. For such reasons, the previous facets of GAs may be regarded as a first order model of human innovation-see Figure 1.

Traditionally, brainstorming has been greatly used as a method to generate a large number of ideas by variety of each participant's knowledge. However, brainstorming does not always work well because of following limitations. At first, brainstorming has a spatial limitation. The spatial limitation forces participants to gather at a same place and a same time. Brainstorming also has an expertise limitation. The expertise limitation blocks participant's group dynamics since different knowledge and culture sometimes make difficulty of communication. Finally, there is a scalability limitation in brainstorming. Human beings cannot communicate too many people at the same time. The scenario gets more convoluted we those experiments are conducted on electronic or online media [10]. HBGAs can help eliminating some of these problems.

\subsection{Brainstorming}

The origin of brainstorming is the 1954 publication of Osborn's "Applied Imagination [15]". He introduced brainstorming as a method, which enables groups to generate more ideas than same number of individuals working separately because of synergy. However, subsequent studies showed some problems in brainstorming such as evaluation apprehension, and production blocking $[1,2]$. The evaluation apprehension tends to occur for low-status members of groups including high-status members. In the situation, low-status members withhold their ideas for fear of suffering negative reactions from high-status members. The production blocking occurs when a participant cannot express his/her ideas because of someone's talking. While listening to others' ideas, the participant may forget his/her ideas.

To solve the problems, electronic brainstorming (EBS) was developed [4]. In EBS, members in a group type ideas anonymously and individually. The anonymity solves the evaluation apprehension, and the individual work solves the production blocking. All participants can access group ideas whenever they want. According to [4], EBS generated triple number of ideas than conventional brainstorming when a number of participants are twelve.

Santanen et al. [16] and Hender et al. [9] have explored effects of stimuli in EBS. According to [9], there are four categories of stimuli:

1. traditional brainstorming (stimuli 1)

2. directory related to the problem (stimuli 2)

3. having associations to the problem (stimuli 3)

4. unrelated to the problem (stimuli 4)

The stimuli 1 are others' ideas. The stimuli 2, for example, are multiple questions that give users to different facets 
of the solution space. The stimuli 2 generated about $133 \%$ quantity of ideas than EBS [16]. The examples of the stimuli 3 are its cause, assumptions, attributes, and so on. The stimuli 3 generated about $120 \%$ quantity and lower quality of ideas than EBS [9]. An example of the stimuli 4 is analogy. The stimuli 4 generated about $80 \%$ quantity and better quality of ideas than EBS [9].

These psychology approaches have improved brainstorming quality and tried to unveil the mystery of human innovation, but we are still in the middle of the puzzle. We still do not know why EBS works well, why analogy works as stimuli, whether or not there is more efficient brainstorming variation, and so on. On the other hand, we already have learned computational innovation from GAs. We believe HBGA-enhanced brainstorming can give us a hint to profoundly understand human innovation.

\subsection{Human-based Genetic Algorithms}

HBGAs outsource GA evolutionary operators to human beings [11]. Examples of HBGAs are Teamwork for a Quality Education (TQE), Free Knowledge Exchange (FKE), and Automatic Concept Generation (ACE) [12, 7, 11, 3].

The TQE project is an application of the basic concepts of genetic algorithms to create a more efficient educational environment $[7,12]$. The participants of TQE are students, faculties and staff advisors. They interact each other according to the principles of GAs. Forty-two students participated a TQE pilot project in 1997, and student feedback was generally good [7].

The FKE project is an evolutionary knowledge management web service [11]. Everyone can voluntarily participate the FKE project. The participants can post problems, post solutions, evaluate the solutions, and modify/recombine the solutions like mutation/crossover in GA operators. More than 500 people from 92 countries attended the FKE project in 7 different languages in 1997 to 2001 [12].

ACE [3] is an email version of FKE. An organizer send a query to the participants, the participants manually apply genetic operators to the query, and reply it. The organizer selects next offspring according to the replies. ACE succeeded to decide the name of itself [3].

Since the HBGAs have performed positive results, we need to step forward for designing a competent HBGA, which enable us to innovate quickly, reliably, and accurately. Elaborate analysis and quantitative evaluation would be helpful to get better understanding of HBGAs and to find a connection between GAs and human innovation. Ueda et al. [19] proposed a methodology for discovering building blocks from text data. Our approach qualitatively evaluates how the structural difference causes HBGA performance.

\section{TWO BRAINSTORMING SETTINGS}

To find a good balance point of human-machine combination in HBGA, we have developed selecto-recombinative brainstorming (SRBS), which is a new brainstorming scheme with enforced idea selection and crossover. To evaluate SRBS quantitatively, we have developed two different discussion components: (1) the SRBS and (2) the threaded discussion board (TDB). Both components were built using the infrastructure provided by the DISCUS project, which is a project to create a distributed and scalable environment for the integration of both human- and computer-generated knowledge in uncertain settings through effective human-human

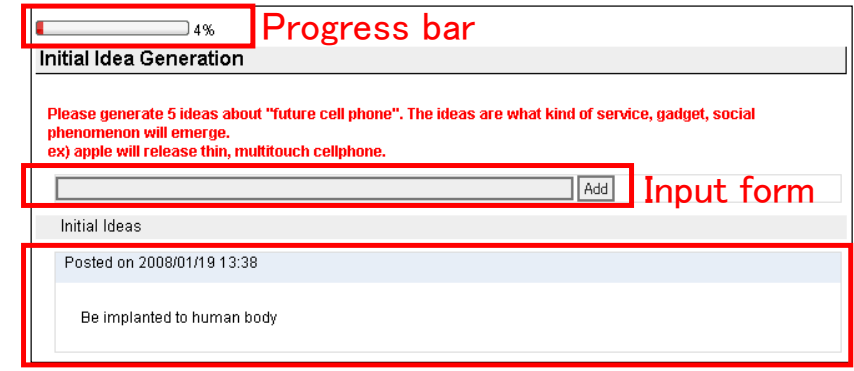

Generated ideas

Figure 2: Initial idea generation in SRBS. When the user inputs his/her idea to the input form and presses the add button, the posted idea appears in the bottom area.

and human-machine collaboration [8]. The DISCUS project provides text processing features (stemming, stop word dictionary, morphological analysis, etc), analysis features (message length enumerator, new term enumerator, KEE [20], social network analysis, etc), and visualization features (bar chart, plot chart, line chart, social network graph, etc). We used the flexibility and the features to make two discussion components, and to analyze the brainstorming on the components.

\subsection{Selecto-Recombinative Brainstorming}

Selecto-recombinative brainstorming (SRBS) is more structured HBGA than threaded discussion boards (TDB). SRBS has three phases, which let the participants in brainstorming process specific tasks, and the phases are initial idea generation, idea selection, and idea crossover.

In the initial idea generation phase, each participant generates five ideas, which are related to an arbitrary theme. Figure 2 shows a web interface on the initial idea generation phase. The user inputs his/her idea to the input form, and presses the add button to post the idea. Then, the posted idea appears in the bottom area. In this phase, each participant can only see their own ideas, but cannot see other participants' ideas. However, each participant can know how many ideas are posted through the progress bar placed in the top area. If the progress bar represents 100\%, which means all participants have finished their five idea submissions, they move to the next phase.

The next phase is an idea selection phase. Each user individually votes for five ideas, which the user prefers, in previously generated ideas. Figure 3 shows a web interface on the idea selection phase. The interface shows all previously generated ideas to the user, and each idea has a vote button. If the user clicks the button, the interface removes the voted idea, and summarizes voting results in the background. After all participants finished their vote, the progress bar indicates $100 \%$, and SRBS passes top ten voted ideas to the next idea crossover phase.

In the idea crossover phase, the participants associate new ideas from the combination of the previously selected ideas. SRBS shows ${ }_{10} C_{2}=45$ combinations of ten selected ideas in previous selection phases to the user. The user generates a new idea, which is related to each the combination. Figure 4 shows the web interface on the idea crossover phase. The user fills the input form with new idea, which is related 


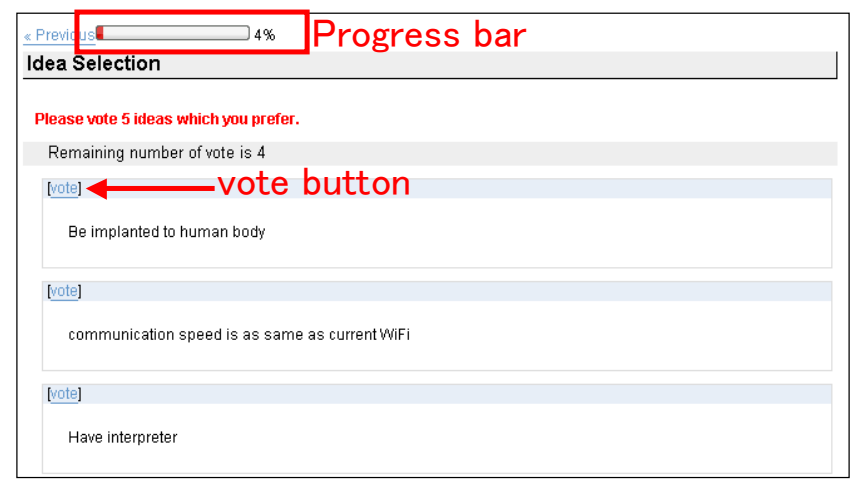

Figure 3: Idea selection in SRBS. The user votes to five ideas by clicking the vote buttons.

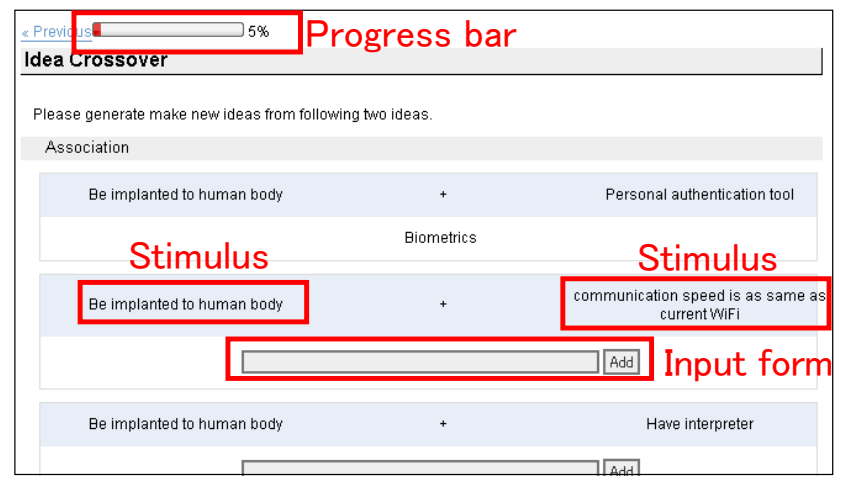

Figure 4: Idea crossover in SBRS. The user inputs a new idea, which is the combination of the stimuli, to the input form.

to two stimuli, and clicks the add button to post the idea. When a total number of generated ideas reach to twenty, the progress bar indicates $100 \%$, SRBS passes the ideas to the next idea selection phase, and participants proceed to the next phase.

The idea crossover and the idea selection will continue until a sufficient number of ideas being generated. SRBS has many magic numbers: making "five" ideas in the initial idea generation, top "ten" ideas in the idea selection, associating "twenty" ideas in the idea crossover, and so on. The numbers are empirically derived, but under study.

As a simple example of how SRBS works, we consider a brainstorming of "future cell phone". This example is based on an actual experiment using SRBS. Three participants, Alice, Bob and Carol, attend the brainstorming.

In the initial idea generation phase, each participant generated two ideas. Alice created:

- Personal authentication tool

- Communication speed is as same as current WiFi

Bob created:

- Be implanted to human body

- Have interpreters

Carol created:
- Can find easily if we lost it

- Not need to bother remaining power.

Next is an idea selection phase. Each participant voted for two ideas, which the participant thought it is good. Table 3.1 represents the voting result. According to the voting results, the next generation is selected as

- Be implanted to human body

- Personal authentication tool

- Communication speed is as same as current WiFi

- Not need to bother remaining power

In the next idea crossover phase, each participant creates new ideas, which is related to the combinations of the previously selected ideas. Alice created:

- Be implanted to human body + Not need to bother remaining power $=$ Drive using energy of human body

- Communication speed is as same as current WiFi + Not need to bother remaining power $=$ Embed cellphone module to all devices

Bob created:

- Not need to bother remaining power + Communication speed is as same as current $\mathrm{WiFi}=$ Standardization of rechargeable system

- Personal authentication tool + Be implanted to human body $=$ Biometrics

Carol created:

- Personal authentication tool + Be implanted to human body $=$ Touch and charge

- Be implanted to human body + Communication speed is as same as current $\mathrm{WiFi}=$ Brain-to-brain communication

These ideas move to an idea selection phase, again.

\subsection{Threaded Discussion Board}

Threaded Discussion Board (TDB) is conventional online threaded bulletin boards added some real-time discussion features with Ajax, and the features are user status notification, discussion status notification, and new message highlighting.

Figure 5 shows the screenshot of the TDB. The user status notification is placed on the navigation bar. It notifies

Table 1: Voting results. The popular ideas are selected as the next generation.

\begin{tabular}{c|c}
\hline Idea & Votes \\
\hline \hline Personal authentication tool & 1 \\
Communication speed is as same as current WiFi & 1 \\
Be implanted to human body & 3 \\
Have interpreters & 0 \\
Can find easily if we lost it & 0 \\
Not need to bother remaining power & 1 \\
\hline
\end{tabular}




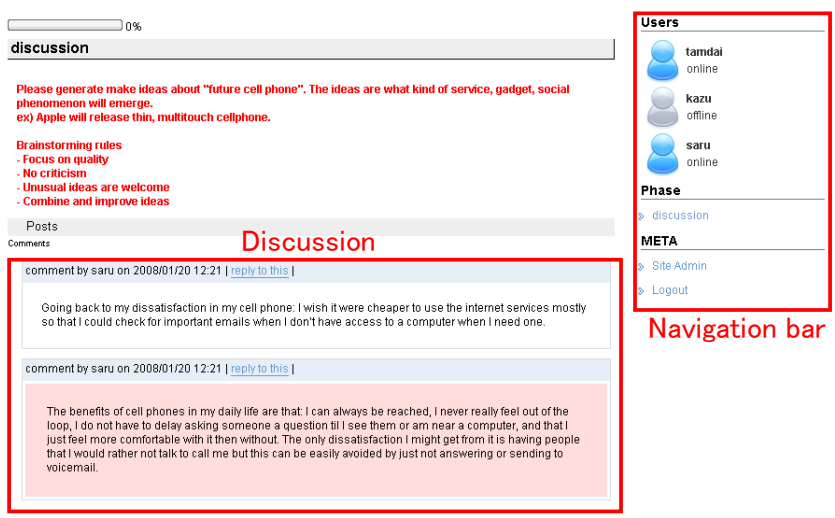

Figure 5: An ongoing brainstorm session on TDB. The navigation bar and the highlighting help the real-time discussion.

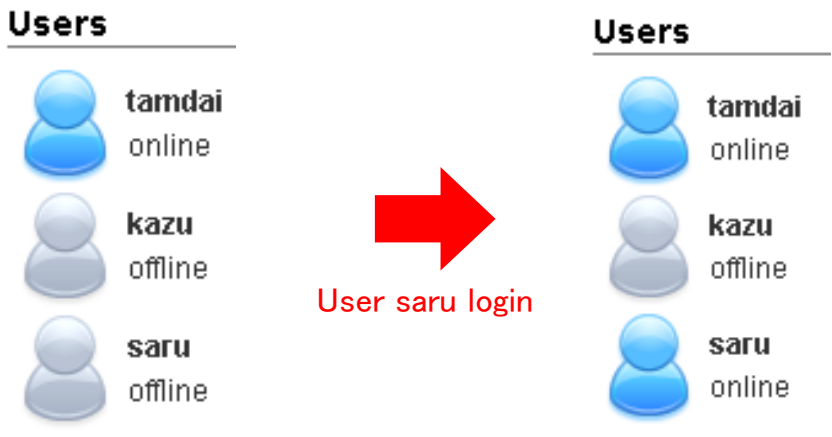

Figure 6: User status notification on TDB. Each participant can know other participants presence with Ajax.

the presence of the participants with Ajax polling (Figure 6 ). The user can know who attend the discussion at the moment. The notification relaxes user's feeling like talking to a wall. The discussion status notification is also placed on the navigation bar. The notification shows a number of new arrived messages with Ajax (Figure 7), and the users can discuss seamlessly. New message highlighting works on the discussion area. It highlights recent five messages. When there are many threads and posts in the discussion, the users have trouble to find new posts. The highlighting helps the users in such a situation.

\subsection{SRBS vs. TDB}

SRBS and TDB have two different facets: user tasks and communication. The user tasks in SRBS are more algorithmic than TDB. SRBS let the users proceed on to three specific tasks: initial idea generation, idea selection, and idea crossover. On the other hand, the users in TDB can freely discuss about their ideas. In spite of both of SRBS and TDB being HBGA, SRBS is closer to GAs than TDB.

The task difference causes communication difference among SRBS and TDB: sparse and dense. In SRBS, the users just need to finish assigned tasks, but cannot talk each other. Since the users share their ideas at transition from one phase to another phase, we can say there is some communication among participants, but it is sparse. On the other hand, the

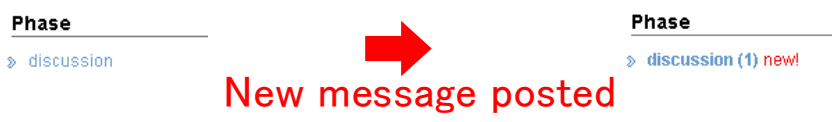

Figure 7: New post notification on TDB. The notification shows a number of new arrived messages with Ajax.

users in TDB can communicate each other whenever they want. We can say there is dense communication in TDB. Following sections describe how the differences influence human creativity process.

SRBS and TDB have one common point: both of them only use other people's idea as stimuli. In SRBS, the users make new ideas with other people's ideas in an idea crossover phase. The users in TDB might sometimes come up with new ideas when the users read other people's ideas in the discussion. Both of SRBS and TDB do not use other extra stimuli.

\section{EXPERIMENTAL SETUP}

To evaluate creativity difference in SRBS and TDB, one brainstorming scenario "what is future cell phone" was used for this experiment. Ten engineering graduate students at the University of Tokyo participated in two five-person brainstorming groups: SRBS and TDB. All students attended the experiment from a web browser with their own PC at different locations, such as their homes, laboratories. The participants were randomly assigned to the groups, and a separate brainstorming activity was started for each group. The participants did not know the identity of their group members. The following instructions were provided to the groups:

Please generate ideas about "future cell phone". The ideas are what kind of service, gadget, and social phenomenon will emerge.

ex) Apple will release an extrathin multitouch cellphone.

Additionally, the brainstorming rules [15] were provided to only TDB group:

- Focus on quality

- No criticism

- Unusual ideas are welcome

- Combine and improve ideas

Each group brainstormed for a period of 40 minutes in Japanese.

\section{RESULTS}

We demonstrate evaluation of HBGA according to settings by comparing SRBS and TDB. We first verify creativity of SRBS and TDB with counting a number of unique ideas in both of them. To count the number of unique ideas, we manually remove duplicated ideas from that same group and off-topic comments. A total number of ideas at each time are shown in figure 8. As shown in the figure, SRBS acquired 3 times more ideas than TDB.

To figure out why SRBS can achieve such high performance, we compared the brainstormings deeply. SRBS and 


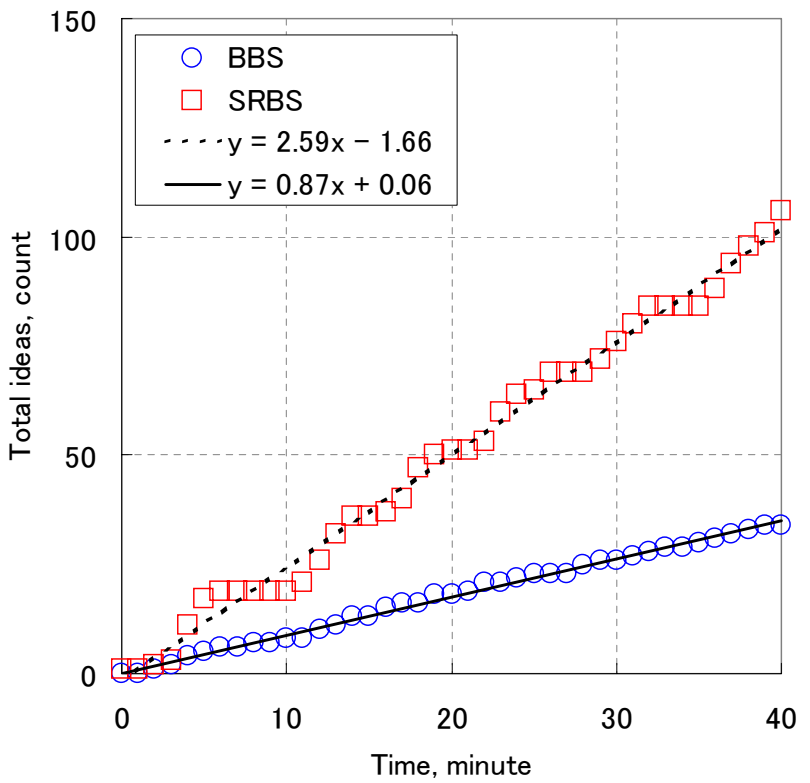

Figure 8: A total number of ideas by time-line. SRBS can acquire up to 3 times more ideas than TDB in same time period.

TDB have the same total message length and a total number of new terms as shown in figure 9 and 10 . The total message length is a total number of characters contained in whole posts at each moment. The total number of new terms is a number of unique terms contained in whole posts at each moment. The terms are extracted with morphological analysis built in DISCUS. Among SRBS and TDB, there are not big difference in the total message length and a number of new terms.

Here we define a divergence ratio:

$$
r_{\text {divergence }}=\frac{n}{l}
$$

where $n$ is the total number of new terms, and $l$ is the total message length. The divergence ratio represents how divergent is the discussion. A divergent discussion makes more new terms than a convergent discussion. The SRBS divergence ratio is:

$$
r_{\text {divergence, } S R B S}=\frac{7.79 t}{53.25 t}=0.146
$$

where $t$ is time. The TDB divergence ratio is:

$$
r_{\text {divergence } T D B}=\frac{6.32 t}{45.96 t}=0.138 \text {. }
$$

Therefore, the divergence ratio of SRBS and TDB are almost the same:

$$
r_{\text {divergence, } S R B S} \cong r_{\text {divergence,TDB }} \text {. }
$$

Although SRBS and TDB have almost the same amount of the divergence ratio, why SRBS can generate more ideas than TDB? To clarify this, we also define a chemistry ratio:

$$
r_{\text {chemistry }}=\frac{m}{n}
$$

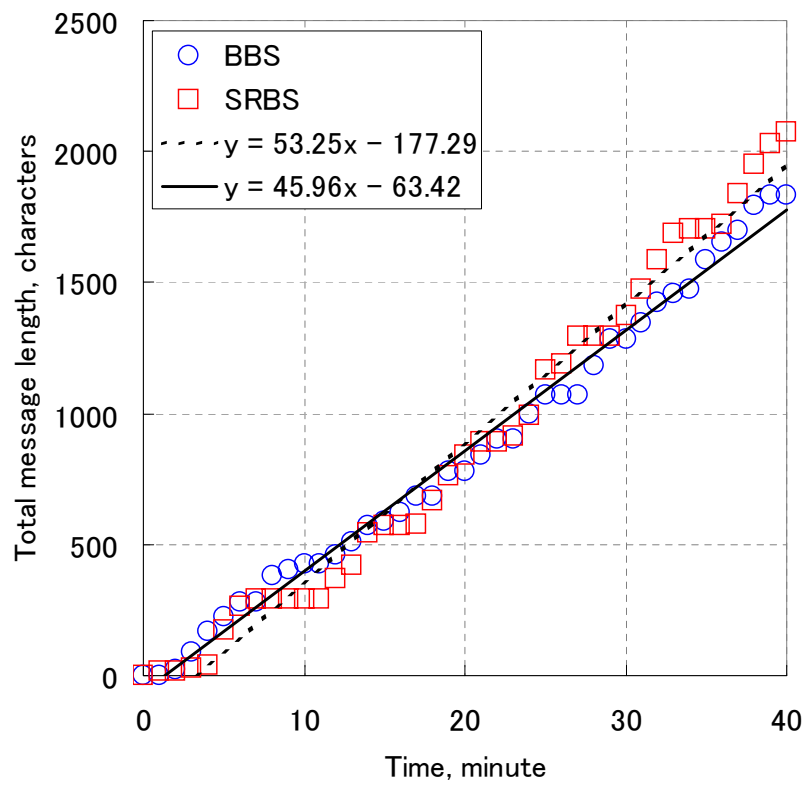

Figure 9: Total message length by time-line. SRBS and TDB have the same message length in same time period.

where $m$ is the total number of ideas, and $n$ is the total number of new terms. The chemistry ratio represents how effectively terms are fused. The SRBS chemistry ratio is:

$$
r_{\text {chemistry }, S R B S}=\frac{2.59 t}{7.79 t}=0.332 \text {. }
$$

The TDB chemistry ratio is:

$$
r_{\text {chemistry }, T D B}=\frac{0.87 t}{6.32 t}=0.138 \text {. }
$$

Therefore, SRBS fuses terms more effectively than TDB:

$$
r_{\text {chemistry, SRBS }}>r_{\text {chemistry,TDB. }} \text {. }
$$

We also examine the user activity difference between SRBS and TDB. A total number of posts are quite different among SRBS and TDB as shown in figure 11. SRBS has 2.6 times more posts than TDS in same time period. Figure 12 shows message length of each post and average message length in before-and-after 5 minutes. SRBS has shorter average message length of each post than TDB. Hence, SRBS shortens each message length and each message posting cycle. The result represents SRBS eliminates superficiality of brainstorming.

Additionally, SRBS can also be improved. SRBS used $38 \%$ of time for idea selection as shown in figure 13. Previous studies on modeling user preferences to speedup interactive genetic algorithms have shown to slice up to seven times the amount of time users spent evaluating solutions. A detailed description is beyond the scope of this paper. A detailed description of such a technique (active interactive genetic algorithms) can be found elsewhere [14].

\section{CONCLUSIONS \& FUTURE WORK}

We proposed a new brainstorming scheme called as selectorecombinative brainstorming (SRBS), which enforces idea 


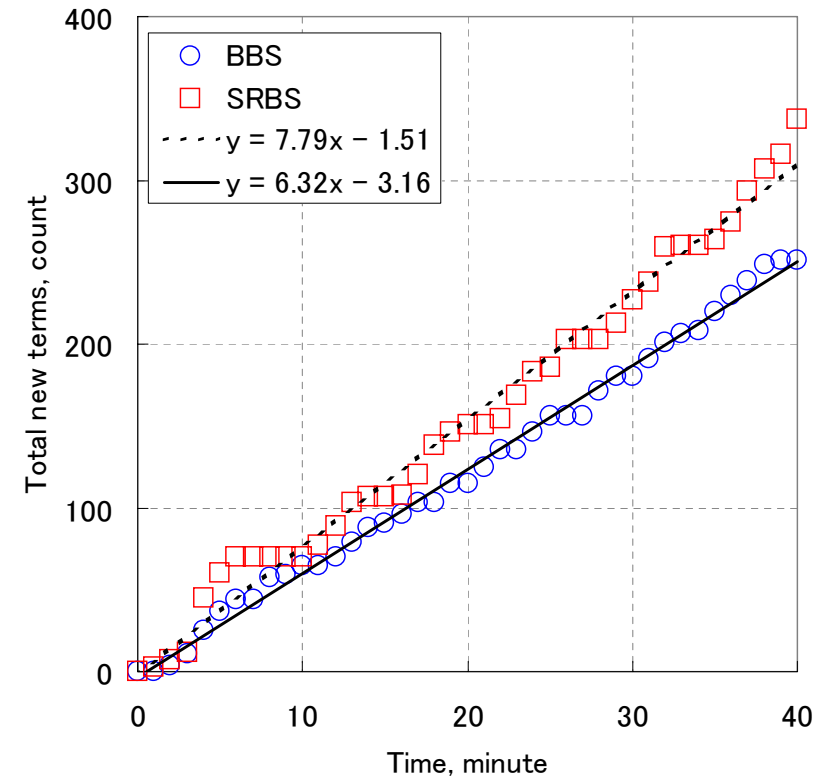

Figure 10: A total number of new terms by timeline. SRBS and TDB have almost the same number of new terms in same time period.

selection and crossover on the users. We quantitatively compared the SRBS with brainstorming on conventional online threaded discussion board (TBD). By modeling creative effort using selecto-recombination, SRBS can provide three times more ideas than TDB.

We defined a divergence ratio and a chemistry ratio to figure out why SRBS works well. The divergence ratio represents how divergent the discussion is, and the chemistry ratio represents how effectively terms are fused in the discussion. Our analysis described SRBS and TDB have almost the same amount of the divergence ratio, but SRBS has a higher chemistry ratio than TDB. We also showed SRBS eliminates superficiality of discussion with SRBS having shorter posting cycle and message length than TDB.

Our next step can be three ways. First, as shown in section 5, SRBS can get more speed because SRBS used $38 \%$ of time for idea selection and the time can be reduced. Second, we can devise stimuli for idea crossover. SRBS in this paper only used other people's ideas as stimuli like traditional brainstorming. However, other stimuli, such as analogy, might increase idea quality and quantity as described in [9]. Finally, we should investigate scalability of SRBS. This paper evaluated SRBS on only a five-person group. Of course traditional face-to-face brainstorming does not work at 100-person groups, but SRBS might be able to exceed the group size limit with certain techniques as GAs could [17].

\section{REFERENCES}

[1] M. Diehl and W. Stroebe. Productivity loss in brainstorming groups: Toward the solution of a riddle. Journal of Personality and Social Psychology, 53(3):497-509, 1987.

[2] M. Diehl and W. Stroebe. Productivity loss in idea-generating groups: Tracking down the blocking

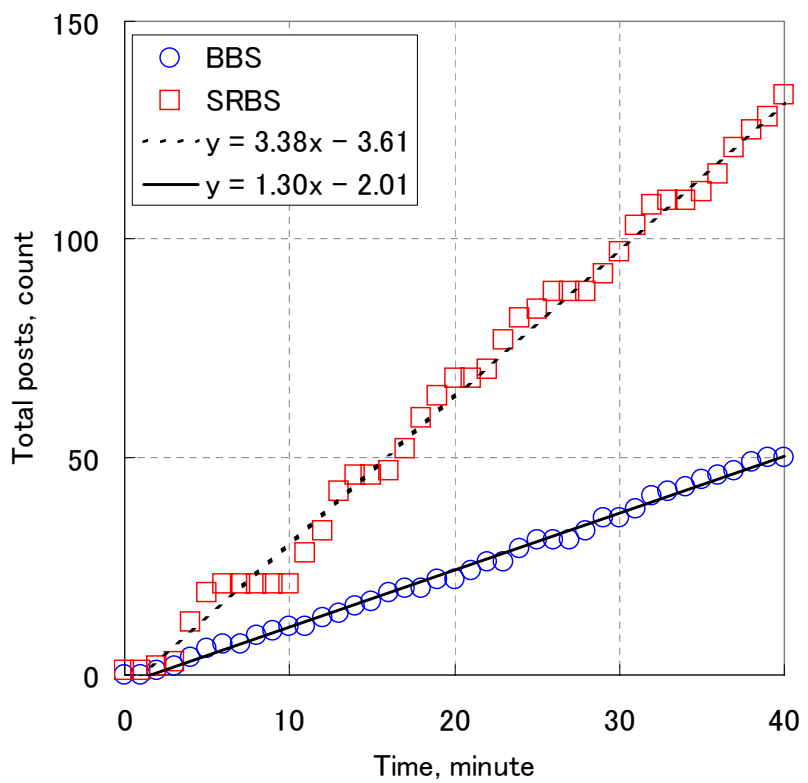

Figure 11: A total number of posts by time-line. SRBS has 2.6 times more posts than TDB in same time period.

effect. Journal of personality and social psychology, 61(3):392-403, 1991.

[3] T. C. Fogarty. Automatic concept evolution (ACE). In Proceedings of Genetic and Evolutionary Computation Conference 2005 (GECCO'05), Washington D. C., June 2005. Late Breaking Paper.

[4] R. B. Gallupe, A. R. Dennis, W. H. Cooper, J. S. Valacich, L. M. Bastianutti, and J. F. N. Jr. Electronic brainstorming and group size. The Academy of Management Journal, 35(2):350-369, 1992.

[5] D. E. Goldberg. Computer-aided gas pipeline operation using genetic algorithms and rule learning. $\mathrm{PhD}$ thesis, University of Michigan. Ann Arbor, MI, 1983.

[6] D. E. Goldberg. The design of innovation: Lessons from and for competent genetic algorithms. Kluwe Academic Publisher, 2002.

[7] D. E. Goldberg, W. B. Hall, L. Krussow, E. Lee, and A. Walker. Teamwork for a quality education: low-cost, effective educational reform, through a department-wide competition of teams. Technical Report 98005, IlliGAL, 1998.

[8] D. E. Goldberg, M. Welge, and X. Llorà. Discus: Distributed Innovation and Scalable Collaboration In Uncertain Settings. Technical Report 2003017, IlliGAL, June 2003.

[9] J. M. Hender, D. L. Dean, T. L. Rodgers, and J. F. N. Jr. An examination of the impact of stimuli type and gss structure on creativity: Brainstorming versus non-brainstorming techniques in a gss environemnt. Journal of Management Information Systems, 18(4):59-85, 2002.

[10] K. Holt. Brainstorming-from classics to electronics. Engineering Design, 7(1):77-82, 1996. 


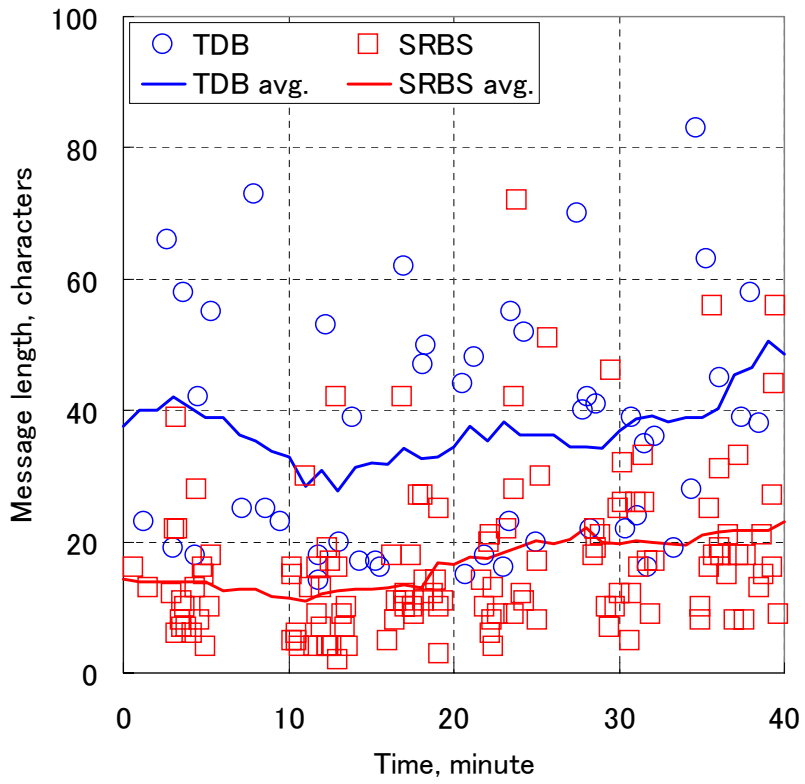

Figure 12: Message length per message and moving average message length in before-and-after five minutes. The $\mathrm{x}$-axis is a moment, on which the message is posted. The $y$-axis is a number of characters, which the message has. SRBS has shorter average message length than TDB.

[11] A. Kosorukoff. Human based genetic algorithm. Technical Report 2001004, IlliGAL, 2001.

[12] A. Kosorukoff and D. Goldberg. Evolutionary computation as a form of organization. In Proceedings of Genetic and Evolutionary Computation Conference 2002 (GECCO'02), pages 965-972, New York, July 2002.

[13] X. Llorà, D. E. Goldberg, Y. Ohsawa, N. Matsumura, Y. Washida, H. Tamura, M. Yoshikawa, M. Welge, L. Auvil, D. Searsmith, K. Ohnishi, and C.-J. Chao. Innovation and Creativity Support via Chance Discovery, Genetic Algorithms, and Data Mining. Journal of New Mathematics and Natural Computation, 2(1):85-100, 2006.

[14] X. Llorà, K. Sastry, D. E. Goldberg, A. Gupta, and L. Lakshmi. Combating user fatigue in iGAs: Partial ordering, support vector machines, and synthetic fitness. In Proceedings of Genetic and Evolutionary Computation Conference 2005 (GECCO'05), pages 1363-1370, Washington DC, USA, June 2005. ACM.

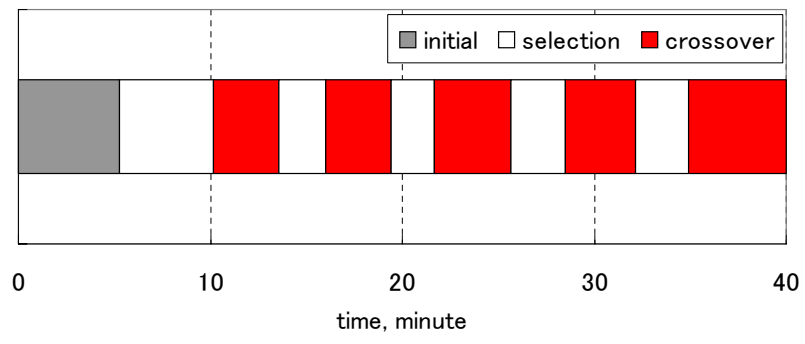

Figure 13: SRBS time-line. 38\% of SRBS time was spent for idea selection.

[15] A. F. Osborn. Applied Imagination: Principles and Procedures of Creative Thinking. Charles Scribner's Sons, 1957.

[16] E. L. Santanen, R. O. Briggs, and G.-J. D. Vreede. Casual relationships in creative problem solving: Comparing facilitation interventions for ideation. Journal of Management Information Systems, 20(4):167-197, 2004.

[17] K. Sastry, D. E. Goldberg, and X. Llora. Towards billion-bit optimization via a parallel estimation of distribution algorithm. In Proceedings of Genetic and Evolutionary Computation Conference 2007 (GECCO'07), pages 577-584, New York, NY, USA, 2007. ACM.

[18] H. Takagi. Interactive evolutionary computation: Fusion of the capabilities of EC optimization and human evaluation. In Proceedings of the IEEE, volume 89, pages 1275-1296, September 2001.

[19] T. Ueda, N. I. Yasui, X. Llorà, K. Sastry, and D. Goldberg. Discovering building blocks for human based genetic algorithms. In Proceedings of Artificial Neural Networks in Engineering 2007 (ANNIE'07), Saint Louis, Missouri, November 2007.

[20] N. I. Yasui, X. Llorà, D. E. Goldberg, Y. Washida, and H. Tamura. Delineating topic and discussant transitions in online collaborative environments. In Proceedings of 9th International Conference on Enterprise Information Systems (ICEIS'O7), pages 14-21, Funchal, Portugal, June 2007. 